\title{
Monitoring of Geographically Distributed Moving Objects
}

\author{
Dr. Ghazi H. Shakah", ${ }^{1, *}$ Vector Kras nopros hin ${ }^{2}$, Aleks ander Valvachev ${ }^{3}$ \\ ${ }^{1}$ Dean of the Faculty of Computer and Information Technology, Ajloun National University, Jordan \\ ${ }^{2}$ Dept. Information Management Systems, Belarusian State University, Minsk, Belarus \\ ${ }^{3}$ Dept. of Information Management Sy stems, Belarusian State University, Minsk, Belarus
}

\begin{abstract}
The paper dwells on how to build monitoring systems for industrial moving objects on the basis of the multi-agent approach and image recognition techniques. The problem of monitoring for diverse business applications is considered. Basic ontological models of the monitoring participants were built, an algorith $m$ to evaluate the object states and generate proper controls was suggested. Five key processes realizing monitoring were distinguished. The architecture for corresponding program agents was suggested together with schematics of their interaction to imp lement the monitoring processes in the Internet environment.
\end{abstract}

Keywo rds Monitoring, Agent Approach, Image Recognition, Monitoring Systems

\section{Introduction}

The quality of human life is determined by the quality of the surrounding environmental conditions. In order to reveal negative changes in the environment and find the ways of their elimination monitoring is used.

In its conventional mean ing "monitoring is a comprehens ive process of observation, evaluation and prediction of changes in the biosphere conditions resulting from natural and anthropogenic factors[1]". Depending on the scope of observations monitoring can be divided into global, regional, and local. As a rule, monitoring involves three basic components: observation of the objects, evaluation of their condition by the given attributes and generation of the adequate control decision.

The latest 100 years have seen the development of monitoring technologies in the frame of ecological research. Special importance was attached to monitoring in the period of ato mic tests (1945-1963) as it helped to minimize negative impact of the radioactive fall-out on the people. Papers such as E.Odum, M.Budyko, M.Begom, J.Harper, S.Pimm, Y.Israel, and others are devoted to the monitoring techniques and approaches.

Conventionally stationary objects have been the targets for monitoring (wild life sanctuaries, natural-territorial habitats, testing grounds, etc.). In general this kind of monitoring was a part of some current task with a few of diagnostic

* Corresponding author:

warsaw2000@yahoo.com (Dr. Ghazi H. Shakah)

Published online at http://journal.sapub.org/ac

Copyright (C) 2011 Scientific \& Academic Publishing. All Rights Reserved indicators, changed occasionally[2].

In the recent years the term monitoring is referred to social, military, logistic, and other issues. Correspondingly monitoring is understood as a package of measures including observing, measuring, evaluating, and decision-making to support the management of natural and anthropogenic systems. The problems have become more sophisticated, whereas the time available to find solutions is little in the opinion of many scientists.

Changing boundaries, market globalization, intensified competition, and labor internationalization together lead to redistribution of energy resources. The number of mobile industrial structures (further-objects), potentially hazardous for the environment, have swollen up respectively. These objects include, first of all, the rolling stock, high-capacity motor transport, and water craft (tankers and dry-cargo freighters) carrying petrochemicals, liquefied gas, wastes of nuclear power plants, and similar products. The traffic of this kind of objects has been intensifying and their number is increasing all over the globe every year. The experience of the recent years proves that despite of all of the precautions taken, accidents and disasters are inevitable. As a result we have plenty negative effects on the human health and life, namely smoke pollution, gas and harmful chemical contamination of densely populated areas and agricultural lands.

To prevent such situations from happening and provide immed iate response in cases of emergency it is necessary, in the opinion of the authors, to work out the techniques and technologies for permanent monitoring of potentially hazardous mobile technical systems. Rapid development of the global network - the Internet as well as inexpensive highly efficient communication and global positioning 
equipment provides a favorable opportunity to find a solution.

The objective of this paper is to study possible ways of building a computer architecture intended for set up and support of automated monitoring of the geographically scattered mobile technical systems (objects).

\section{Setting the Objectives}

When setting the objectives for this study the authors took into consideration the need for representation of multiple realistic cases, therefore the objectives and the solution can be considered in a certain sense typical for monitoring [3].

The given conditions: the company $\mathrm{W}$, including the control center $\mathrm{H}$, and $\mathrm{n}$ of mobile objects to be managed $\mathrm{G}=$ $\mathrm{G} 1, \mathrm{G} 2, \ldots, \mathrm{Gn}$. The objects (for instance a diesel unit and $\mathrm{n}$ of cistern cars) are participating in a certain mission (for example, transporting petroleum). The success of the mission depends on the state of the objects [4].

The state of the objects $G$ is characterized by a finite number of diagnostic variables:

$$
\mathrm{X}=\left(\mathrm{X}_{1}, \mathrm{X}_{2}, \ldots, \mathrm{X}_{\mathrm{m}}\right)
$$

The $\mathrm{G}$ objects are geographically scattered and change their geographical positions $\mathrm{K}^{\mathrm{t}} \mathrm{G}$ with time:

$$
\mathrm{K}^{\mathrm{t} 1} \mathrm{G} \neq \mathrm{K}^{\mathrm{t} 2} \mathrm{G}
$$

In order to evaluate the current state of the objects the W company management should periodically meet the challenge of monitoring $\mathrm{M}$, which implies evaluation of the state (V) of the objects $(\mathrm{G})$ based on the current values of the variables $\mathrm{X}$, as well as synthesis of the proper control decision (U):

$$
\mathrm{M} \rightarrow \mathrm{U}=\mathrm{f}(\mathrm{X}, \mathrm{V})
$$

The need is to develop the system architecture Sys that would provide automated solution for the problem of monitoring $\mathrm{M}$.

The requirement to the solution: invariance of the monitoring time to the number of objects $(t \rightarrow 0$ with $n \rightarrow \infty)$.

We shall accept the following considerations as the basis for the solution:

the feature of the problem set is the geographical distribution of the objects and, correspondingly, scattered nature of the information on their state. In the majority of cases the multi-agent approach[5] is used to solve this kind of problems;

as per the process approach[6] the solution of the problem can be reduced to five processes, namely building the company $\mathrm{W}$, acquiring information $\mathrm{X}$ from the objects, transmitting $X$ to the center, integrating $X$ into the central $\mathrm{DB}$, processing $\mathrm{X}$ to evaluate $\mathrm{V}$ of the object and generate $\mathrm{a}$ proper control decision $U$.

Comparing the results of the agent and process approaches we can make the following first conclusion: the architecture Sys must include five agents, all of which will contribute to the monitoring solution embracing five processes mentioned above.

\section{Key Models}

In order to create a theoretical bas is for the solution a set of models is suggested grounded by the ontological approach[3], promoted in the Belarusian State University of Informatics and Radioelectronics and Belarusian State University[5-7].

Ontology is a formal specification of the divisible conceptual model[3]. Ontology involves content classes of object regions, properties of these classes, links between the classes, and affirmations, made of these classes, their properties and links between them. In what follows we shall name ontological models simply "models" for convenience.

In compliance with the logics of the monitoring problem, first of all it is necessary to build a model of the company, which will perform mon itoring.

The company model must include at least the attributes of the center and objects:

$$
\mathrm{W}=(\mathrm{H}, \mathrm{G} 1, \mathrm{G} 2, \ldots, \mathrm{Gn})
$$

The model of the center contains the global identification attributes, list of monitoring objectives, a server, processing software $\mathrm{F}$ for indicators $\mathrm{X}$, and the database:

$$
\mathrm{H}=\left(\mathrm{A}^{\mathrm{H}}, \mathrm{idH}, \text { task, } \mathrm{S}, \mathrm{PL}, \mathrm{DB}\right)
$$

where: $\mathrm{A}^{\mathrm{H}}$ - is the global address of the center; idH center's identifier; task -the monitoring tasks; $\mathrm{S}$ - server; PL - programs to synthesize states and controls; DB - is the database to accumulate the data sent by the detectors.

The model for the problem of monitoring synthesis is similar to the classical control problem[4]:

$$
\mathrm{U}=\mathrm{F}(\mathrm{X}, \mathrm{V})
$$

where: $\mathrm{F}$ - is the control choice function for the object depending on its state.

The object model involves the components, necessary to identify the object in the Internet, acquire object data, and trans mit it to the center for processing:

$$
\mathrm{G}=\left(\mathrm{A}^{\mathrm{G}}, \mathrm{idG}, \mathrm{KG}, \mathrm{d} 1, \mathrm{~d} 2, \ldots, \mathrm{dm}, \mathrm{Ks}, \text { Box, Kp }\right)
$$

where: $A^{H}$ - is the global address of the object; id $\mathrm{G}$ - the object's identifier; $\mathrm{G}$ - current position data; $\mathrm{d}$ - detectors; $\mathrm{Ks}$ - is the controller to read and analyze the signals from the detectors; $\mathrm{d}$-detectors; Box - a message from the object to the center; $\mathrm{Kp}$ - is the controller for data exchange between the objects and the center.

The model of the package includes the addresses of the sender and recipient, object identifying attributes, and detector readings:

$\operatorname{Box}=\left(\mathrm{A}^{\mathrm{H}}, \mathrm{A}^{\mathrm{G}}, \mathrm{id} \mathrm{G}, \mathrm{X}\right)$

On the whole the models 1-6 are the bas is for the solution. The ontological approach used enables to specify them to the level of the program code. The overall monitoring diagram is plotted in Figure 1.

This diagram is of an open nature and can be extended indefinitely by increasing the number of the objects to be monitored $\mathrm{G}$. The time $t$ required for the control $U$ synthes is is invariant to the number of objects, since the processes to acquire the vector values for diagnostic variables $\mathrm{X}$ have been multi-sequenced. One of the possible alternatives to arrange the agent architecture that implements this scheme is 
given below.

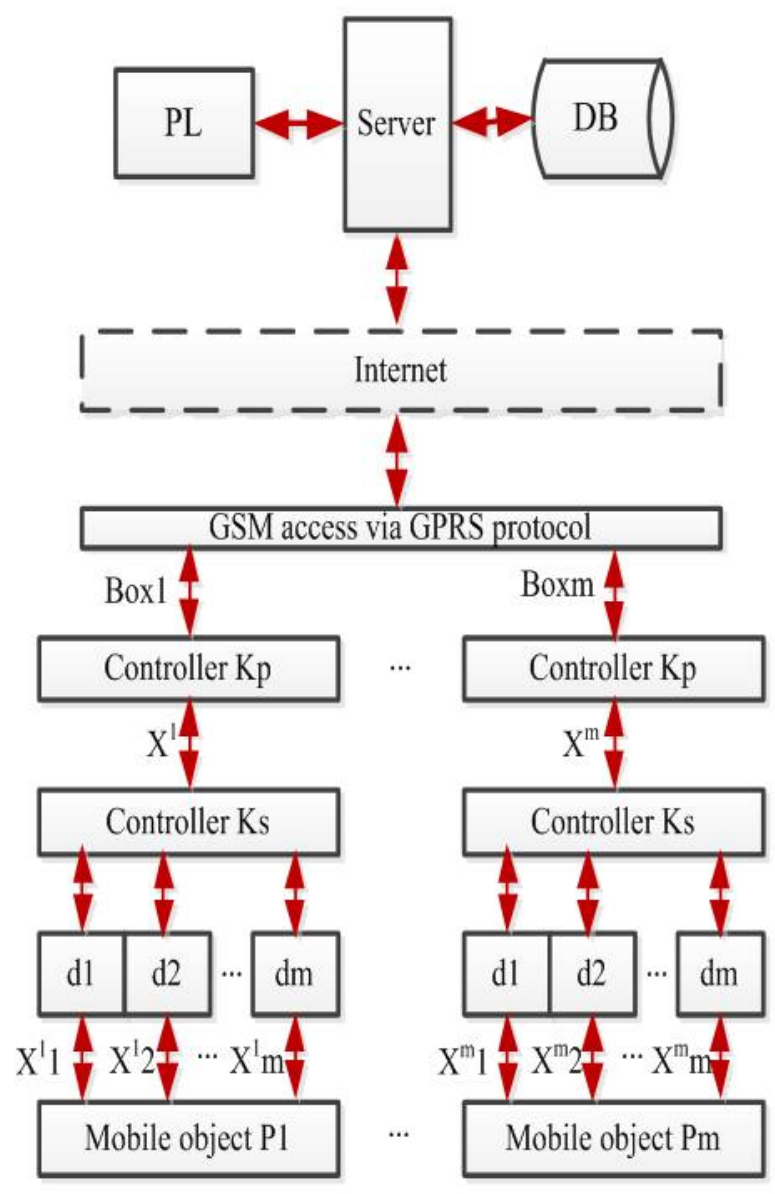

Figure 1. Scheme of monitoring

\section{System Architecture}

The system architecture consists of the modules performing the processes that contribute to the solution. It has been mentioned above that the solution should be reduced down to five processes that would, as a whole, support monitoring; therefore there must be five agents [6, 7].

The processes are synchronized by the company management; thus we shall primarily build the model of the agent that generates the model of the hie rarchy including the attributes of all the animate and virtual (programs, devices) participants (Figure 2).

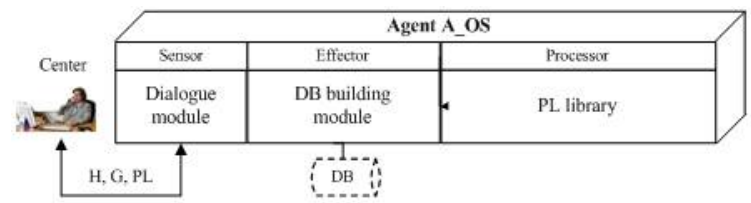

Figure 2. Architecture of the company model agent

Through the dialogue with the company management the sens or module of the agent A_OS acquires all the necessary information both about the center itself and about the objects to be monitored. All the information obtained is accumulated in the database (DB). The data analys is programs are in the library PL, which is generated at the stage of the agent compilation.

The detectors are the primary information source for this type of monitoring. Respectively the second agent must receive the values of the attributes, formalize them, and pass them on to the communication agent. The corresponding architecture is shown in Figure 3.

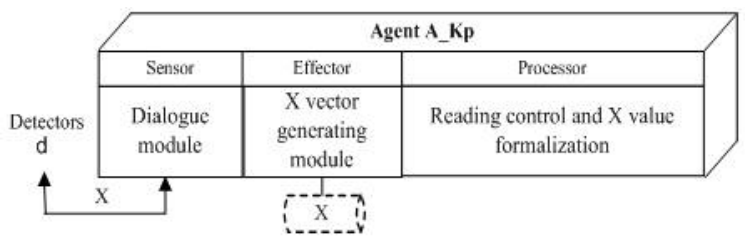

Figure 3. Architecture of the agent to generate the indicator value vector

The agent is run, as a rule, in a discrete mode, namely the indicator values are taken in certain periods of time, preset by the expert while developing the controller. In terms of hardware the agent is represented by a set of detectors and a programmab le controller. The detectors measure $\mathrm{X}$ values of the $\mathrm{X}$ parameters, the controller formalizes them and performs their preprocessing.

Then the agent is activated that receives the $\mathrm{X}$ vector and generates the burst message Box to the center, describing the state of an object. The burst contains the indicator vector and identifying attributes of the object. The agent architecture A_Ks, performing these operations, is shown in Figure 4.

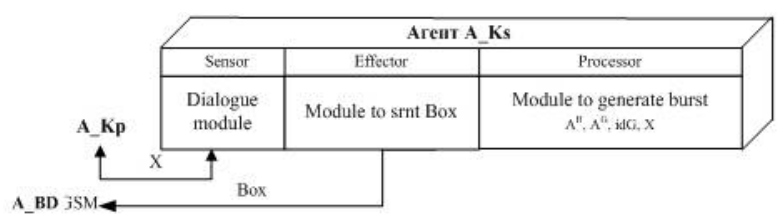

Figure 4. Burst generat ing agent architecture

As soon as the burst has been generated, the agent A_Ks gets the access to the GSM network via the GPRS protocol and sends Box to the central server.

In terms of hardware the agent A_Ks is represented by a programmable controller and interface with external communications.

The functions of obtaining and integrating the burst into the database are fulfilled by the agent A_DB, the architecture of which is illustrated in Figure 5.

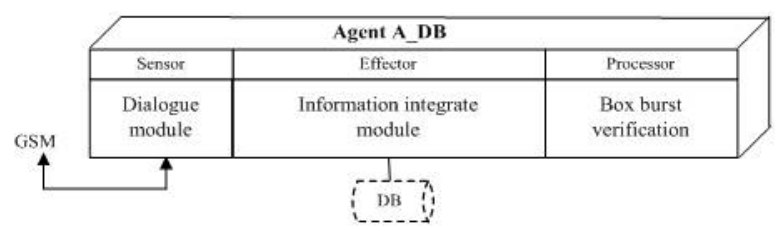

Figure 5. Application agent architecture

The agent analyzes the input information, identifies the task and the object, verifies the $\mathrm{X}$ vector values, performs statistic processing, and integrates the result into the database of the center.

The information integrated into the database, can be used for synthesis of the object states, generating the control, 
prediction, etc. Besides, it can be employed for the OLAP analysis using Data Mining and Knowledge Discovery techniques. The database can be accessed through subscriber dispatcher programs, co mpatib le with such Internet brows ers as MS Explorer, Opera, FireFox, etc.

The agent A_PL, the architecture of which is shown in Figure 6, is used for processing of the user database inquires.

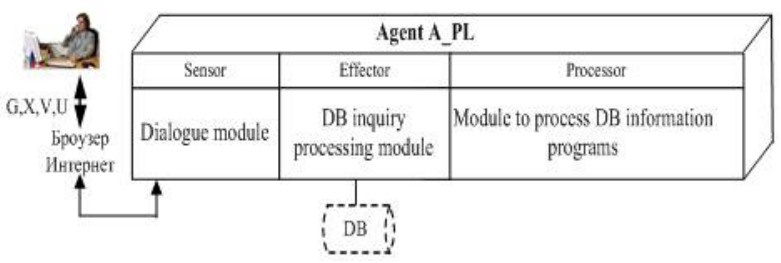

Figure 6. Architecture of the inquiry processing and applicat ion agent

The server resources (database and processing software package) can be accessed as soon as the input user code has been verified. The use of publicly available browsers makes it possible to control the state of remote objects from stationary or portable PCs, co mmunicators, pads, etc. The PL library has a modular structure thus the list of active tasks can be constantly extended.

Therefore the monitoring system architecture can be represented as an aggregate of five program agents, a server, a database, and standard Internet dialogue and communication means and digital telephony.

This approach enables to upgrade the hardware and software parts of each of the monitoring participants without disturbing the others. If the communication requirements have been changed reprogramming the controller in the A_Ks agent will suffice.

Figure $7 \mathrm{p}$ lots an example of the overall arch itecture based on the developed approach for remote monitoring of diesel units.

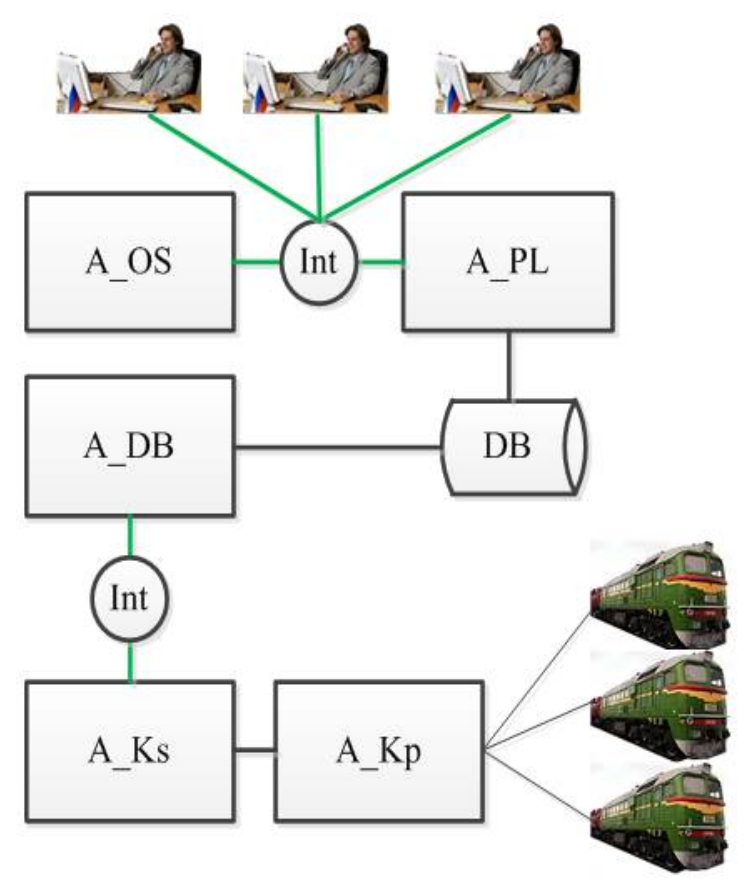

Figure 7. Monitoring system architecture
A wide range of detectors and programmable controllers has been developed for practical implementation of similar architectures. Currently the detectors are capable of taking values of over 20 different parameters (temperature, vibration, pressure, fuel level in tanks, engine revolutions, currents, subscriber positions in GPS, GLONASS, etc.), typical of mobile mechanisms (diesel units, traction units, cars, trucks, tankers, etc.). The programming option account for fast adjustment of the controllers for a new object domain and selection of the detector configuration.

The key unsolved problem that increases the cost of this alternative of the system architecture is an extremely labor-intensive customization of the processing and PL library programs to work with a certain object domain. In addition the number of products in case of logical output of the state V of objects Gdepending of values X, as well as the synthesis of controls $\mathrm{U}$ depending on $\mathrm{V}$, can reach several thousands, which impedes not only the program debugging but also understanding the product array logics by an unprepared user.

\section{Conclusions}

This paper has discussed the issues related to building flexible software-hardware architectures to arrange and support monitoring of geographically scattered mobile objects.

A broad concept for monitoring has been suggested on the basis of the synthesizing the process and multi-agent approaches, and digital communication and web-interface technologies.

The problem of monitoring for diverse business applications is considered. Basic ontological models of the monitoring participants were built, an algorithm to evaluate the object states and generate proper controls was suggested. Five key processes realizing mon itoring were distinguished.

The architecture for corresponding program agents was suggested together with schematics of their interaction to implement the monitoring processes in the Internet environment.

\section{REFERENCES}

[1] V. E. Sokolov, Regional Ecological Monitoring. M.: Science, 1983. $-263 \mathrm{p}$

[2] D. Lindenmayer, G. Likens, Effective Ecological Monitoring. 5 Earthscan. $184 \mathrm{p}$

[3] C. Caero, F. Ruiz, Ontologies for Software Engineering and Software Technology. Springer. $-339 \mathrm{p}$

[4] W. L. Brogan, Modern Control Theory. Prentice Hall. $736 \mathrm{p}$

[5] V. V. Krasnoproshin, Knowledge-Based Systems: Problems and Solutions, Proceedings of the International Conference 
on Modeling and Simulation (MS'2006). Konya, Turkey, 2006. Vol. 1. P. 1-4

[6] G. Shakah, V. V. Krasnoproshin, A. N. Valvachev, Decision Making System for Operative Tasks . Proceedings of 10-th
International Conference (PRIP'2009), Minsk, May 19-21, 2009. Минск, 2009. Р. 272-275

[7] M. Wooldridge Multiagent Systems. - John Wiley \& Sons. 2002. - 340 p 